\section{Adverse Reactions to Drugs}

Many modern drugs are extremely potent and useful, but often the margin between the therapeutic dose and one which causes adverse reactions is small. Greater skill is needed in using these drugs to the best advantage than was required to prescribe the elegant placebos of the past. Adverse reactions range from minor symptoms such as a dry mouth or sleepiness up to potentially fatal damage to the bone marrow or the liver. It is important to detect and report adverse reactions so that the problem can be recognized and brought to the attention of the profession by the medical journals and the Committee on Safety of Drugs.

Two techniques have been used to collect information about adverse reactions. Doctors have been asked to report them to a central body by use of a simple printed form. An example is the yellow warning postcard issued in the United Kingdom by the Committee on Safety of Drugs. Unfortunately, only a small proportion of adverse reactions get reported when this technique is used. At Johns Hopkins Hospital, in Baltimore, the number of reports received increased fourfold when a programme of active surveillance replaced one which relied on the resident staff completing a drug reaction card attached to the notes. The second method is for the adverse reactions to be sought by a doctor or nurse who makes regular visits to patients and inquires specifically about adverse reactions. As might be expected, this produces a much higher return.

Three articles in this week's B.M.f. report a study of this second method carried out in Belfast, and show that drug reactions occurred in $10.2 \%$ of 1,160 patients who received drug therapy. The figure may seem high, but figures of $15 \%$ and $18 \%$ have been reported from similar surveys carried out in the Johns Hopkins Hospital ${ }^{1}$ and Montreal General Hospital. $^{2}$ Studies which have relied upon completion of adverse reaction reports have given figures of only $1-3 \% .^{34}$

The number of adverse reactions to drugs depends partly upon their toxicity and partly on the frequency with which they are prescribed. It is unwise to assume that one penicillin or phenothiazine is more toxic than another without knowing the relative amounts which are used. Even in hospital it may be difficult to obtain an accurate record of the drugs administered to each patient. ${ }^{.5}$ and in the community-where patients may be receiving tablets from more than one source-the problems are obviously much greater.

Fortunately, most of the reactions are not severe, and in the Belfast survey only four threatened life. Most studies have shown that reactions occur predominantly in the first few days of hospital admission, when sick patients are riost likely to be treated with drugs. In Montreal $87 \%$ of the adverse reactions were due to the main pharmacological action of the drug, and half of these resulted from overdosage. The majority of adverse reactions are caused by a relatively small number of drugs, with digitalis and antibiotics heading the list.

The incidence of adverse reactions to drugs rises with the number of different drugs prescribed to each patient. In the Belfast study two-thirds of the patients who were in hospital

\footnotetext{
1 Seidl, L. G., Thornton, G. F., and Kluff, L. E., Amer. f. publ. Hlth, $1965,55,1170$

2 Ogilvie, R. I., and Ruedy, J., Canad. med. Ass. F., 1967, 97, 1450.

${ }^{3}$ McDonald, M. G., and MacKay, B. R., f. Amer. med. Ass., 1964, 190, 1071.

4 Seidl, L. G., Friend, D., and Sadusk, J., f. Amer. med. Ass., 1966, 196, 421.

${ }^{5}$ Crooks, J., et al., Lancet, 1967, 1, 668.
}

for more than three weeks had received six or more different drugs. A study of adverse reactions to methicillin carried out in the United States ${ }^{1}$ showed that on average each patient had received 14 other drugs during his stay in hospital. It would be helpful to establish which combinations are particularly likely to be hazardous and try to improve understanding of the mechanisms involved. In Belfast the combination of digitalis and diuretics was a potent source of adverse reactions.

There are some important lessons to be learnt from these surveys of unwanted and potentially hazardous drug reactions. The frequency of these reactions enjoins caution on all who prescribe drugs-particularly those such as digitalis, anticoagulants, and antidiabetic and antihypertensive agents whose dose regulation is critical. Nevertheless, it is reassuring to find that many reactions are not severe, and many doctors might not consider some nausea with digitalis or looseness of the bowel with ampicillin worth reporting as an adverse reaction. Active surveillance of adverse reactions to drugs involves a full-time commitment for one or more doctors. Expense alone would rule out such techniques being generally applied, but a few carefully conducted surveys of this kind on a continuing basis should be valuable in detecting changes in the pattern of adverse reactions to drugs.

\section{Of Voles and Men}

In January 1965 a mongol patient from Calderstones Hospital was found to be suffering from tuberculosis of the skin. ${ }^{1}$ The disease affected the part of his arm on which he had been vaccinated 15 years previously during a trial organized by the Medical Research Council to test the protection produced by B.C.G. and to compare it with that offered by the vole bacillus. $^{2}$ The subjects had been schoolchildren in the Birmingham and Manchester areas and the inmates of a number of institutions, which included Calderstones Hospital and Brockhall Hospital in North-east Lancashire.

Alerted by the discovery of this case, Dr. Anne Maguire inspected the 110 survivors of the 180 inmates originally vaccinated at these two hospitals, and she found 16 more patients who were similarly affected. All the lesions had occurred at the sites of the inoculations, and histologically they were tuberculoid granulomas in which tubercle bacilli could not be seen. This is the usual state of affairs in lupus vulgaris. Though the vole bacillus could not be grown in culture or recovered by inoculation of voles, the circumstances under which the cases had occurred, the strongly positive Mantoux status of the patients, and the response of the lesions to antituberculous treatment can leave little doubt that the vole bacillus was responsible. Mnguire proposed the name lupus murinus for this disease. Apart from a variable degree of regional lymphadenopathy no other evidence of tuberculosis could be found in any of the patients.

The vole bacillus was discovered in 1937 by A. Q. Wells, ${ }^{3}$ who had recently gone to Oxford with an M.R.C. grant $^{4}$ to collaborate with C. E. Elton in studying the cause of recurrent fluctuations in the vole population that were believed to result from some kind of infection. The mycobacterium that he isolated from a naturally infected field vole Microtus agrestis from Scotland proved to $\mathrm{be}^{5}$ the cause of endemic 
tuberculosis in this species, and also in the bank vole Clethrionomys glareolus, the wood mouse Apodemus sylvaticus, and the shrew Sorex araneus. The disease, which was widely spread in Britain, caused a chronic inflammation, particularly in the subcutaneous tissues and the lymphatic system ; but it proved not to be the cause of the fluctuations in vole population that had been the original object of the study.

Clinical interest now centred upon the possible use of the vole bacillus for protecting man against tuberculosis, for it appeared that the vole bacillus was not pathogenic for man, though it caused the Mantoux reaction to become positive. This organism was thought to be a form of Mycobacterium tuberculosis, though there were certain differences-for example, its growth was inhibited in culture media containing glycerin, and it presented curved or hooked forms, especially when seen in the tissues. The war interrupted the development of this idea, but by 1946 Wells $^{5}$ felt in a position to say that he thought the vole bacillus was incapable of producing other than localized disease, and that a large scale trial comparing it with B.C.G. was justifiable. The trial $^{2}$ was mounted in 1949 by the Medical Research Council, B.C.G. and vole bacillus vaccines being compared. Wells inoculated the patients at the two hospitals in question ${ }^{1}$ in May 1950. The vaccine was administered by a multiple puncture technique in order to avoid the troublesome ulcers." that usually followed subcutaneous injection.

Wells died in 1956, and it seems that interest in the vole bacillus died with him. His last recorded opinion of the vaccine $^{6}$ recognized that lupus occurred rather more frequently after it than it did after B.C.G. and that local lymphadenopathy was somewhat more troublesome. But he seems to have considered that the vole bacillus was at least as good a prophylactic against tuberculosis as B.C.G., and that it had the theoretical advantage of being an organism that existed in its natural state of virulence and was not, like B.C.G., artificially attenuated, with the consequent twin dangers of eventually losing useful antigenic properties or of reverting to a more virulent phase. (It is not suggested that this latter eventuality is more than a theoretical possibility, the major disaster at Lübeck in 1935 having been attributed to contamination of the vaccine. ${ }^{7}$ ) Incidentally, Wells was critical of the Czechoslovakian work with the vole bacillus, in which an organism was used that had been attenuated by prolonged culture on artificial media, whereas he had been careful to maintain the virulence of his own vaccine by repeated passage back. into voles.

Maguire's discovery raises two points upon which it is interesting to speculate. Firstly, an incidence of 17 cases of lupus murinus among 110 patients vaccinated is much higher than the $3 \%$ reported $^{2}$ in the M.R.C. trial, and it suggests that it would be well worth while to review as many of the subjects of the original trial as possible. L. J. Witts, ${ }^{4}$ in his obituary on Wells, mentions that extensive trials of the vole bacillus vaccine were also being made in Rhodesia, and further information might be forthcoming from this quarter. Secondly,

1 Maguire, A., Brit. f. Derm., 1968, 80, 419.

2 D'Arcy Hart, P., Pollock, T. M., Sutherland, I., Bibl. tuberc. (Basel), 1957, 8, 171.

S Wells, A. Q., Lancet, 1937, 1, 1221.

4 Witts, L. J., F. Path. Bact., 1958, 75, 495 5 Wells, A. Q., M.R.C. Special Report, No. 259, 1946. London,

6 Wells, A. Q., Bibl. tuberc. (Basel), 1957, 8, 61.

- Rosenthal, S. R., B.C.G. Vaccination Against Tuberculosis, 1957. Boston, Little, Brown.

${ }^{8}$ Horwitz, O., Meyer, J., Bibl. tuberc. (Basel), 8, 245. there is no evidence to suggest that the vole bacillus has caused anything more than local disease, whereas B.C.G. may very rarely cause generalized, even fatal, tuberculosis." If the vole bacillus is really incapable of causing generalized disease might it not be worth while to reconsider its use as a prophylactic, particularly as lupus murinus is treatable? It might be possible to modify the lupus-producing proclivities of the vaccine, either by controlling the dose, by analogy with the observations of O. Horwitz and J. Meyer ${ }^{8}$ in B.C.G. lupus, or by modifying the technique of vaccination, as suggested by P. D'Arcy Hart and his colleagues. ${ }^{2}$ In fact this outbreak of lupus murinus, far from constituting an absolute contraindication to the use of the vole bacillus, as might seem to be the first obvious conclusion, might really be seen as an opportunity for reconsidering the value of the vole bacillus as a prophylactic agent that produces a purely. local infection. There is no doubt, however, that Wells considered a living vaccine, however good it might be, as second best should a killed vaccine ever become a practical possibility.

\section{Jakob-Creutzfeldt Syndrome : an Infective Disease?}

Increasing interest in the so-called "slow virus" diseases of the nervous system has raised the possibility that certain obscure and as yet unexplained diseases of the brain and spinal cord may be of similar aetiology. Kuru, a progressive disorder characterized by increasing cerebellar ataxia and dementia, which occurs in the Fore tribe in New Guinea, can be transmitted to chimpanzees when material from affected human brains is inoculated ${ }^{1}$ into them. Work is now in progress to find out whether there is any possibility that commoner human diseases such as multiple sclerosis and motor neurone disease could also prove to be due to transmissible agents.

The term "Jakob-Creutzfeldt disease" has been widely and erroneously used by neurologists and physicians in Britain for many years to identify a syndrome, often familial, in which features of Parkinsonism are aocompanied by progressive dementia, signs of corticospinal tract degeneration, and sometimes peripheral amyotrophy due to anterior horn cell disease. Such a clinical picture, evolving over a period of some years, is not uncommon, and resembles the so-called Parkinsonism-dementia complex which has been found to occur frequently in the Mariana Islands in the Pacific. Such cases occurring sporadically or in more than one member of a family in western Europe and in the United States are probably better described as examples of corticostriatonigral degeneration.

The condition which A. Jakob and H. G. Creutzfeldt originally described is a disorder characterized by dementia of comparatively rapid progression accompanied by frequent myaclonic jerking and other less constant findings, often

1 Gajdusek, D. C., Gibbs, C. J., and Alpers, M., Science, 1967, 155, 212.

= Brownell, B., and Oppenheimer, D. R., F. Neurol. Neurosurg. Psychiat., 1965, 28, 350.

3 Nevin, S., McMenemey, W. H., Behrman, S., and Jones, D. P., Brain, $1960,83,519$.

4 Gibbs, C. J., et al., Science, 1968, 161, 388. 